\title{
Noncontact technique for measuring surface tension and viscosity of molten materials using high temperature electrostatic levitation
}

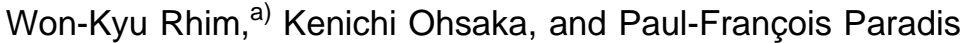 \\ Jet Propulsion Laboratory, California Institute of Technology, Pasadena, California 91109 \\ R. Erik Spjut \\ Department of Engineering, Harvey Mudd College, Claremont, California 91740
}

(Received 10 February 1997; accepted for publication 22 February 1999)

\begin{abstract}
A new, noncontact technique is described which entails simultaneous measurements of the surface tension and the dynamic viscosity of molten materials. In this technique, four steps were performed to achieve the results: (1) a small sample of material was levitated and melted in a high vacuum using a high temperature electrostatic levitator, (2) the resonant oscillation of the drop was induced by applying a low level ac electric field pulse at the drop of resonance frequency, (3) the transient signals which followed the pulses were recorded, and (4) both the surface tension and the viscosity were extracted from the signal. The validity of this technique was demonstrated using a molten tin and a zirconium sample. In zirconium, the measurements could be extended to undercooled states by as much as $300 \mathrm{~K}$. This technique may be used for both molten metallic alloys and semiconductors. (C) 1999 American Institute of Physics. [S0034-6748(99)00206-3]
\end{abstract}

\section{INTRODUCTION}

Surface tension and the viscosity of molten metals and semiconductors, two important parameters in the numerical modeling of various industrial processes (such as casting, welding, melt spinning, and crystal growth processes), have always been limited when dealing with high temperature materials. Conventional methods used to measure surface tension are the sessile-drop method, the pendant-drop method, and the maximum bubble pressure method, while commonly used methods of measuring viscosity are the capillary method, the oscillating vessel method, the rotational method, and the oscillating-plate method. ${ }^{1}$ Although these techniques are adequate for chemically inert liquids, they are not for many high temperature materials which may react strongly with their containers. For this reason and others, such as equipment, calibration, data treatment, etc., the data available regarding high temperature materials tend to show wide scatter. In a given measurement method, disagreement among surface tension data of a same sample material can be caused by different surface conditions. The viscosity may be less sensitive to surface contamination than the surface tension. However, the viscosity data are particularly sensitive to flows in liquids which might be caused by external perturbation forces such as the gravity force, the applied magnetic forces, or the electrostatic forces.

While these conventional approaches are hampered by sample contamination, the levitation methods are not. One of the advantages of the levitation method is that it isolates samples from crucibles. Furthermore, if the levitated sample is processed in a high vacuum, it is also protected from surrounding gases. For this reason electromagnetic levitators

\footnotetext{
a) Author to whom all correspondence should be addressed; electronic mail: won-kyu.rhim@jpl.nasa.gov
}

(EMLs) have frequently been used for surface tension measurements of conducting materials. In the earlier EML experiments, ${ }^{2-4}$ Rayleigh's equation, ${ }^{5}$ which assumes a spherical drop shape, was used to extract surface tension from observed resonance frequency. In reality, however, a drop levitated in an EML is nonspherical. The result is that the spectrum of an oscillating drop often reveals three to five closely spaced peaks. Cummings and Blackburn ${ }^{6}$ have analyzed this and related the experimentally observed spectrum with Rayleigh frequency. However, some of drawbacks associated with EML are that the sample materials have to be electric conductors, and it cannot measure the viscosity primarily due to the drop instability and internal flow induced by the rf field that is needed to levitate a sample.

In this article we introduce a new noncontact method which enables us to measure the surface tension and the viscosity. This technique was developed on the basis of the high temperature electrostatic levitator (HTESL), ${ }^{7}$ utilizing its unique capabilities. The result is a technique which measures both surface tension and viscosity in the following environment: (i) HTESL levitates a charged sample in a high vacuum using computer controlled electrostatic fields, (ii) it can process materials such as metals, semiconductors, and some insulators, (iii) sample temperature can be varied over a wide range, and samples may be deeply undercooled, (iv) the shape of a levitated melt is approximately spherical, and the effect of surface tension due to a small deviation from sphericity and nonspherical surface charge distribution can be corrected according to the correction term which was derived by Feng and Beard, ${ }^{8}$ (v) both the surface tension and the viscosity may be obtained from a single transient signal, and (vi) a single axisymmetric mode can be excited, making the data analysis rather straightforward and unambiguous. 


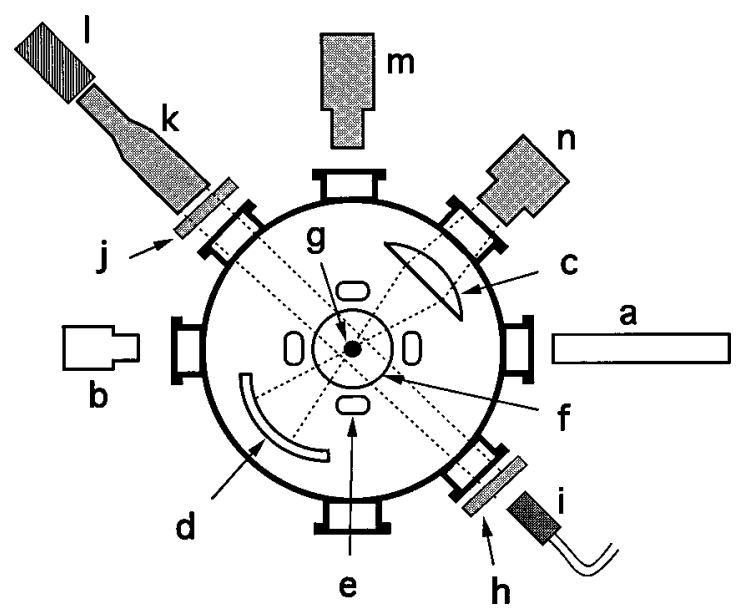

FIG. 1. Schematic diagram of the high temperature electrostatic levitator at the JPL: (a) He-Ne position sensing laser, (b) position sensing detector, (c) focusing lens, (d) focusing reflector, (e) side positioning electrode, (f) top/ bottom electrodes, (g) sample, (h) back light diffuser, (i) fiber optical back light, (j) He-Ne blocking filter, (k) long distance microscope, (l) charge coupled device (CCD) camera, (m) pyrometer, (n) xenon heating lamp or YAG laser.

\section{THEORETICAL BACKGROUND}

An isolated liquid drop (free from external force) assumes a spherical shape due to the uniform surface tension. If the drop undergoes a small amplitude axisymmetric oscillation, the drop shape $r(t)$, in a weak damping limit, can be described by

$$
r(t)=r_{0}+\sum_{n=2}^{\infty} r_{n} \cos \left(\omega_{n} t\right) P_{n}(\cos \theta) \exp \left(\frac{-t}{\tau_{n}}\right),
$$

where $r_{0}$ is the radius of the drop when it assumes a spherical shape, $P_{n}(\cos \theta)$ is the $n$th order Legendre polynomial, $\theta$ is the angle measured between the $z$ axis and the radial direction, and $r_{n}$ is the oscillation amplitude of the $n$th mode. The $n=1$ term was omitted in the equation since it only describes translational movement of the drop. The characteristic oscillation frequency $\omega_{n}$ corresponding to the $n$th mode is given $b^{5}$

$$
\omega_{n}^{2}=n(n-1)(n+2) \frac{\sigma}{\rho r_{0}^{3}},
$$

where $\sigma$ is the surface tension and $\rho$ is the density of the drop. The damping constant $\tau_{n}$ in Eq. (1) is given by

$$
\frac{1}{\tau_{n}}=(n-1)(2 n+1) \frac{\eta}{\rho r_{0}^{2}},
$$

where $\eta$ is the viscosity of the liquid sample. ${ }^{9}$ For $n=2$, the drop shows axisymmetric oscillations as shown in Fig. 1 at a frequency

$$
\omega_{2}^{2}=\frac{8 \sigma}{\rho r_{0}^{3}},
$$

and the oscillation will decay with the time constant

$$
\frac{1}{\tau_{2}}=\frac{5 \eta}{\rho r_{0}^{2}}
$$

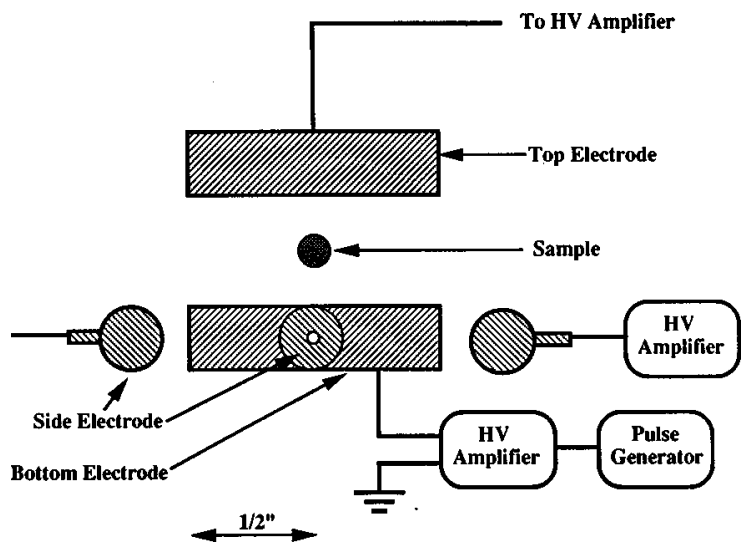

FIG. 2. Schematic of the electrode arrangement by which a drop oscillation can be induced.

If a drop carries a uniformly distributed surface charge $Q_{s}$, the resonant oscillation frequencies are modified by it. From an energy stability analysis, Rayleigh ${ }^{10}$ first calculated the characteristic frequencies for small-amplitude oscillations of a charged drop in the absence of external fields, and established the amount of charge necessary to induce disruption of the drop surface. According to Rayleigh, once the drop charge $Q_{s}$ is determined, the oscillation frequency $\omega_{2}$ of the $P_{2}(\cos \theta)$ mode is given by

$$
\omega_{2 c}^{2}=\frac{8 \sigma}{\rho r_{0}^{3}}\left(1-\frac{Q_{s}^{2}}{64 \pi^{2} r_{0}^{3} \sigma \epsilon_{0}}\right),
$$

where $\omega_{2 c}=2 \pi \nu_{2 c}$ is the angular frequency of a charged drop oscillation, $\rho$ is the liquid density, and $\epsilon_{0}$ is the permittivity of vacuum. From Eq. (6), the surface tension, $\sigma$, can be expressed explicitly as

$$
\sigma=\frac{r_{0}^{3} \rho}{8}\left(\omega_{2 c}^{2}+\frac{Q_{s}^{2}}{8 \pi^{2} r_{0}^{6} \rho \epsilon_{0}}\right) .
$$

If the sample charge can be assumed to be a point charge which is positioned right at the middle of a pair of infinite parallel electrodes, the drop charge $Q_{s}$ in Eq. (6) can be determined by the levitation condition, i.e.,

$$
m g=\frac{Q_{s} V}{L},
$$

where $m$ is the sample mass, $g$ is the gravitational acceleration, $V$ is the potential difference between the top and bottom electrodes, and $L$ is the spacing between the electrodes. In practice the electric field experienced by a sample is affected by the finite size of electrodes and the nonuniform charge distribution on the drop, and therefore, one has to go through more elaborate numerical analysis to determine $Q_{s}$, taking into account the actual electrode arrangement, sample position, sample size, and charge distribution on the drop surface (see Fig. 2).

The shape of a drop which is levitated by an electric force against the gravity force deviates from a perfect sphere regardless of the size and the amount of charge on the drop. Equilibrium shapes and the stability of a charged conducting drop levitated by a uniform electric field were analyzed by Adornato and Brown ${ }^{11}$ whose asymptotic analysis for the 
equilibrium drop shape in small electric fields showed the appearance of second and third Legendre functions. The nature of axisymmetric oscillation of electrostatically levitated drops, however, was examined later by Feng and Beard ${ }^{8}$ using an analytic multiple parameter perturbation method. In the presence of electric and gravitational fields, the smallamplitude oscillation of a charged drop differs from Rayleigh's linear modes. The oscillatory response at each frequency consists of several Legendre polynomials rather than just one, and the characteristic frequency for each axisymmetric mode decreases from that calculated by Rayleigh for stronger applied electric field. Feng and Beard's frequency correction term for axisymmetric $n=2$ mode is given by

$$
\omega_{2 c+}^{2}=\omega_{2 c}^{2}[1-F(\sigma, q, e)],
$$

where

$$
F(\sigma, q, e) \equiv \frac{\left(243.31 \sigma^{2}-63.14 q^{2} \sigma+1.54 q^{4}\right) e^{2}}{176 \sigma^{3}-120 q^{2} \sigma^{2}+27 \sigma q^{4}-2 q^{6}} ;
$$

$\sigma$ is the surface tension, $\omega_{2 c}$ is as defined by Eq. (6), and $q$ and $e$ are defined by

$$
q^{2}=\frac{Q_{s}^{2}}{16 \pi^{2} r_{0}^{2} \epsilon_{0}}
$$

and

$$
e^{2} \equiv E^{2} r_{0} \epsilon_{0},
$$

respectively, where $E$ is the applied electric field.

\section{EXPERIMENTAL ARRANGEMENT AND PROCEDURE}

The technique was developed on the basis of the HTESL at the Jet Propulsion Laboratory (JPL). A schematic diagram of the HTESL is shown in Fig. 1. The HTESL levitates a sample 1-3 $\mathrm{mm}$ in diameter between a pair of parallel disk electrodes spaced about $12 \mathrm{~mm}$ apart (see Fig. 2). The electric field between these two electrodes generates controlled electrostatic forces on a charged sample that cancel the gravitational force and maintain the sample at a preset position, and the four small side electrodes around the bottom electrode control the sample's position in the horizontal plane. The electrode assembly was housed in a stainless steel chamber which was typically evacuated to $\sim 10^{-8}$ Torr before sample heating began. Samples were heated using a 1 $\mathrm{kW}$ xenon arc lamp up to $\sim 1000^{\circ} \mathrm{C}$. At this temperature the xenon arc lamp was gradually switched to a $100 \mathrm{~W}$ YAG laser to reach higher temperature. In the process of measuring the surface tension and viscosity, the sample temperature had to be brought to a predetermined value by controlling the lamp power (or laser power). A pyrometer which operated at a wavelength $4 \mu \mathrm{m}$ was used to avoid any interference by the xenon arc lamp radiation. When only the YAG laser was used, a single color pyrometer was used that operated at 750 $\mathrm{nm}$. Other details about the HTESL are given in an earlier publication. $^{7}$

To excite the drop oscillations a few modifications had to be made to the existing electrostatic levitator. These in-
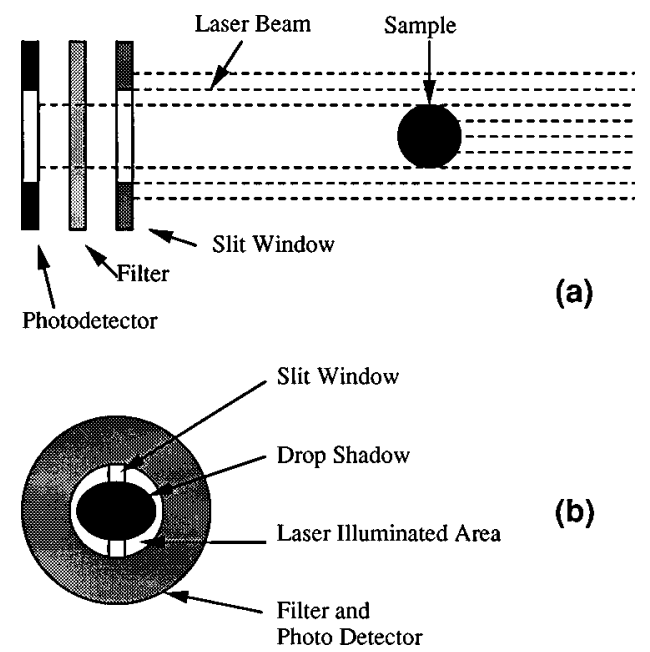

(b)

FIG. 3. Schematic diagram of the drop-amplitude detection system.

cluded the insertion of an ac voltage amplifier between the bottom electrode and the electric ground as schematically shown in Fig. 2. As usual, the top electrode was connected to a high voltage amplifier for levitation control. The electrode assembly, being axially symmetric, could effectively excite even modes in which the $n=2$ mode was the lowest mode. The oscillating amplitude of a drop was detected using the drop-amplitude detection system shown in Fig. 3. The levitated drop was back lit by a collimated laser beam to create a drop shadow on a circular photodetector. The drop shadow was masked from the detector except for a narrow slit so that the detected signal was sensitive to the oscillating drop amplitude. A narrow band interference filter at the laser wavelength was placed in front of the detector to reject stray light. The linearity of the detector was tested by blocking the detector by known step increments.

When dealing with a volatile sample material, the sample has to be weighted before and after the experiment, and the actual sample mass at the time of data taking can be closely estimated using the thermal history during the experiment and the temperature dependence of the vapor pressure of the material.

\section{DROP EXCITATION AND DATA ANALYSIS}

Two computer programs Excite-to-Disk (ETD) and Stored-Data-Analysis (SDA) were developed using the National Instruments Labview system. The first creates an excitation pulse consisting of a given number of sine-wave cycles at a given frequency and amplitude, and it is applied to the lower electrode after amplification. The ensuing transient signal is the recorded time stamped and saved to a disk in a binary (non-ASCII) format. For reference, the high-pass filtered data in the time domain along with the Fast Fourier transform (FFT)-based power spectrum are displayed. The data are assumed to follow the function

$$
y=A e^{-t / \tau} \sin (2 \pi f t+\phi),
$$

where $A$ is the amplitude, $t$ is the time, $\tau$ is the decay time constant, $f$ is the frequency, and $\phi$ is a constant phase factor. The program calculates estimated values for the decay time constant, $\tau$, in seconds, and the frequency, $f$, in $\mathrm{Hz}$, both from the FFT and from the period between zero crossings. 


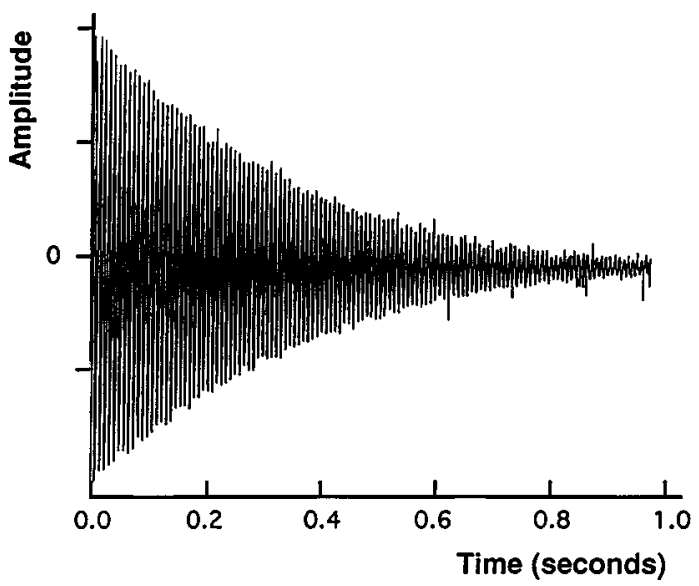

FIG. 4. Typical transient oscillation of a levitated drop. This particular signal is from a molten $\mathrm{Zr}_{76} \mathrm{Ni}_{24}$ sample.

The SDA program reads the binary data file created by the Excite-to-Disk. It permits selection of the relevant portion of the data, and then performs a nonlinear least-squares fit to the function

$$
y=A e^{-t / \tau} \sin \left[2 \pi\left(f_{0}+f_{1} t\right) t+\phi\right],
$$

where the data are assumed to be the product of a decaying exponential and a sine wave whose frequency is allowed to change linearly with time. The reported parameters are the exponential-decay time constant $\tau$ in seconds, the initial frequency $f_{0}$ in $\mathrm{Hz}$, and the coefficient for the variation of frequency in time $f_{1}$ in $\mathrm{Hz} / \mathrm{s}$, as well as the approximate standard deviation for each of these parameters.

The technology demonstration for surface tension measurement was conducted using a molten tin and a molten zirconium sample. Initially the excitation pulse length and amplitude were adjusted to be approximately $0.25 \mathrm{~s}$ and 200 $\mathrm{V}$, respectively, and the drop response was observed as the carrier frequency was varied searching for the $P_{2}(\cos \theta)$ mode of the oscillations. Once the right mode was found, the carrier frequency and the pulse amplitude were reset in the Excite-to-Disk program to make the drop oscillation amplitude approximately $5 \%-10 \%$ of the drop radius. A typical transient signal obtained through such a process is shown in Fig. 4. Equations (6)-(12) were used to determine the surface tension, and Eq. (5) was used to determine the viscosity.

\section{RESULTS AND DISCUSSION}

The results of the surface tension measurements of tin are given in Fig. 5. The linear fit to the data can be expressed by

$$
\begin{aligned}
\sigma(T)= & 541.3-0.09(T-505) \quad \mathrm{mN} \mathrm{m}^{-1} \\
& (493-723 \mathrm{~K}),
\end{aligned}
$$

while the result of Kasama et al. ${ }^{12}$ which was obtained using the sessile-drop method in an argon-hydrogen atmosphere was expressed by

$$
\sigma(T)=560-0.091(T-505) \quad \mathrm{mN} \mathrm{m}^{-1}
$$

$$
\text { (505-1723 K). }
$$

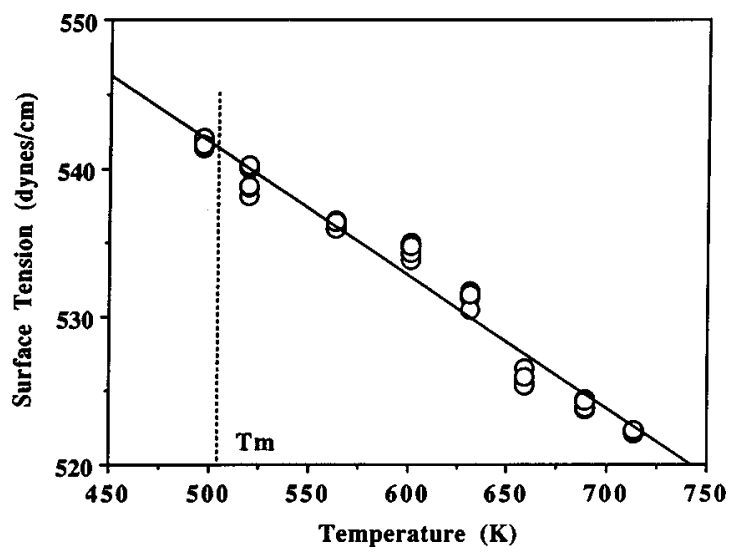

FIG. 5. Surface tension of a tin sample as a function of temperature.

Taking the mean value found in many literature surface tension data, Keene ${ }^{13}$ presented the following relation for tin:

$$
\begin{aligned}
\sigma(T)= & 561.6-0.103(T-505) \mathrm{mN} \mathrm{m}^{-1} \\
& (520-1670 \mathrm{~K}) .
\end{aligned}
$$

Our result is about $3.4 \%$ lower than the literature values. Other literature values for tin surface tension at the melting temperature range between 532 and $622 \mathrm{mN} \mathrm{m}^{-1}$. ${ }^{12}$ The variation in surface tension values is most likely caused by the degree of surface contamination, most likely by tin oxides. Even in our sample, we could observe a few oxide patches floating on the surface of molten drops. Heating the drop several hundred degrees above its melting temperature did not substantially reduce the surface patches. Further evidence of contamination could be seen from the less than 50 $\mathrm{K}$ undercooling. No special attention was given to preparing cleaner tin samples. Judging by the data scattering, for a given sample at a fixed temperature, the surface tension data are reproducable within a $1 \%$ uncertainty.

Our experiment also showed the temperature coefficient to be $-0.09 \mathrm{mN} \mathrm{m}^{-1} \mathrm{~K}^{-1}$ which is in excellent agreement with the $-0.091 \mathrm{mN} \mathrm{m}^{-1} \mathrm{~K}^{-1}$ measured by Kasama et al., however, it is about $13 \%$ smaller than the mean valued provided by Keene. The temperature coefficients which appear in various references range between -0.02 and -0.22 $\mathrm{mN} \mathrm{m}^{-1} \mathrm{~K}^{-1}$. 12

The viscosity data obtained by substituting measured decay time constants $\tau_{2}$ into Eq. (5) are shown in Fig. 6 along with the reference data by Iida $e t a l$. which were obtained using the capillary method. ${ }^{14}$ The solid line is the exponential fit of Iida's result. Our data show the range of data scattering when the measurement was repeated five times at each temperature with the average value shown as a closed circle. The scatter in the tin viscosity was found to be about 0.2 $\mathrm{mPas}$, which corresponds to an $\sim 13 \%$ uncertainty in the viscosity of tin. We believe that this scatter was caused by the position controlling electrostatic field.

As an application of the present technique to a refractory material, we chose a $99.98 \%$ pure zirconium sample obtained from Alfa Chemical. Unlike the tin, the levitated molten $\mathrm{Zr}$ drops showed no oxide patches or any other structures on their surfaces, and they undercooled as much as $300 \mathrm{~K}$ when they were allowed to cool freely. With the melting 


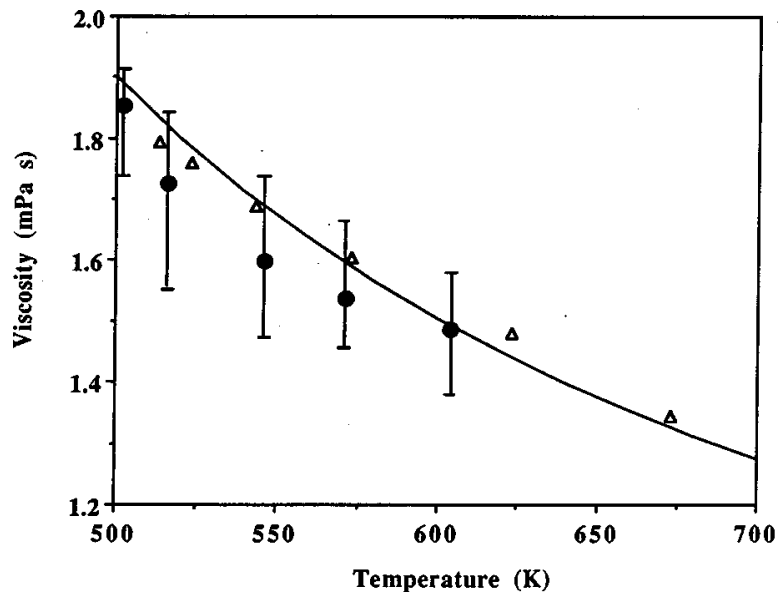

FIG. 6. Viscosity of the same tin sample of Fig. 5 as a function of temperature. The error bars show the spread in the data when repeated five times at each temperature. The closed circle shows the average of five data points. The solid line is the exponential fit to the results of Iida et al. (Ref. 14) (the open triangles).

temperature of zirconium being $2128 \mathrm{~K}$, impurities and zirconium oxides on the sample surface seemed to have been either evaporated or dissolved at high temperature. For the measurements of surface tension and viscosity, the laser power was controlled to obtain a steady state sample temperature before transient oscillations were induced on the drop.

The results for the surface tension and the viscosity of this material are shown in Figs. 7 and 8, respectively. The surface tension data could be expressed by

$$
\sigma(T)=1459-0.244(T-2128) \quad \mathrm{mN} \mathrm{m}^{-1}
$$

$$
(1850-2200 \mathrm{~K}) \text {, }
$$

while the corresponding viscosity data could be fitted by

$$
\eta=4.86-5.3 \times 10^{-3}(T-2128) \quad \mathrm{mPa} \mathrm{s}
$$

over the same temperature range.

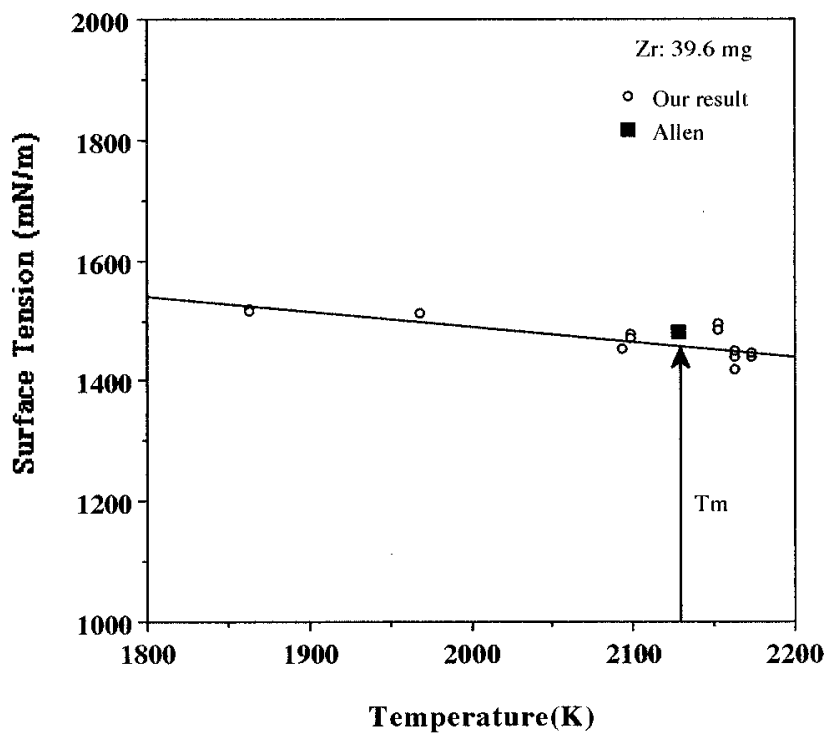

FIG. 7. Surface tension of zirconium as a function of temperature. An undercooled state of almost $300 \mathrm{~K}$ could be reached.

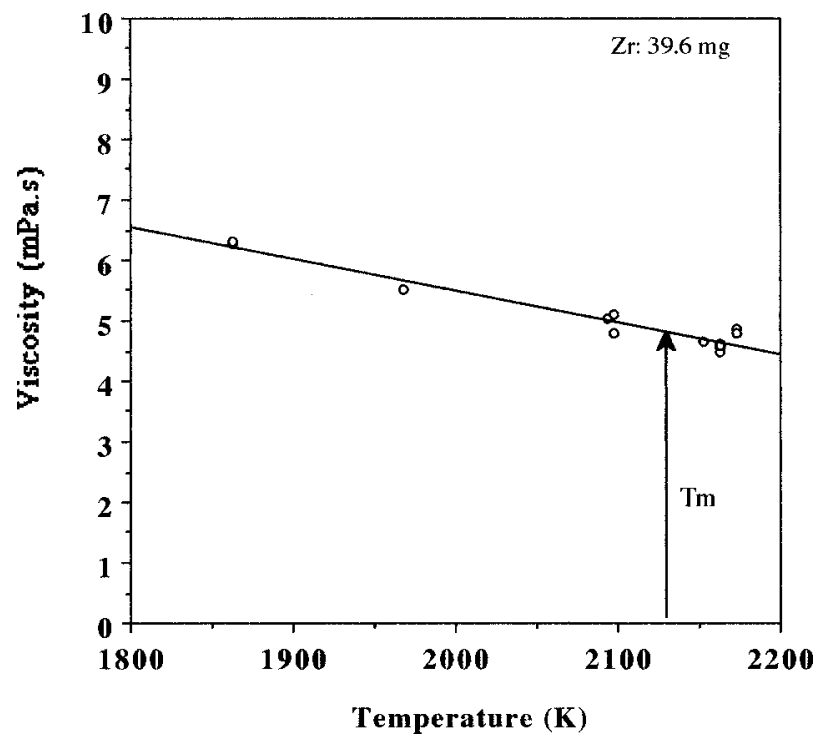

FIG. 8. Viscosity of zirconium as a function of temperature. An undercooled state of almost $300 \mathrm{~K}$ could be reached.

The surface tension value obtained at its melting temperature, $1459 \mathrm{mN} \mathrm{m}^{-1}$, is $1.4 \%$ smaller than Allen's value of $1480 \mathrm{mN} \mathrm{m}^{-1}$. ${ }^{15}$ Frohberg et al. recently used an electromagnetic levitator and measured surface tension of pure zirconium both on a ground based and in a microgravity (micro-g) environment of a space shuttle. ${ }^{16}$ Their ground and micro-g results can be expressed, respectively, by

$$
\begin{gathered}
\sigma_{1-\mathrm{g}}(T)=1543-0.66(T-2128) \mathrm{mN} \mathrm{m}^{-1} \\
(2000-2250 \mathrm{~K})
\end{gathered}
$$

and

$$
\sigma_{\mu-\mathrm{g}}(T)=1512-0.37(T-2128) \quad \mathrm{mN} \mathrm{m}^{-1}
$$

$$
\text { (1950-220 K). }
$$

At the melting temperature, their space result is about $3.6 \%$ larger than ours, and their ground result is larger than ours by $5.7 \%$. Their temperature coefficients are at least $50 \%$ larger than ours. Discrepancies between these surface tension data and the literature values are difficult to explain at this time. However, according to Chen and Overfelt, ${ }^{17}$ the magnetic field, used in electromagnetic levitation, causes a slight increase in the stiffness of the drop, raising the apparent surface tension. This effect may be more pronounced for larger samples since they feel a stronger magnetic pressure. Probably, this would account for the discrepancies.

The viscosity data shown in Fig. 8 are probably the first of its kind measured on molten zirconium. In particular, the data obtained in the deeply undercooled state demonstrate the importance of this technique. About $\pm 0.3 \mathrm{mPa}$ s of uncertainty shown by the data corresponds to about $\pm 6 \%$ of uncertainty in the zirconium viscosity. Unfortunately, no reference could be found to compare with our results.

In summary, the present technique is a noncontact technique based on electrostatic levitation which attempts to simultaneously measure the surface tension and the viscosity from a levitated material. This technique will be applicable to highly reactive molten refractory materials or to deeply 
undercooled liquids. If this technique is used in the Earth's environment, it will be limited to liquids that have viscosities larger than $\sim 1 \mathrm{mPas}$. The uncertainty in the viscosity which was induced by control if the sample position seems to stay at less than $1 \mathrm{mPas}$ in its absolute magnitude.

For liquids having viscosities higher than a certain limit, the present technique may not be able to induce oscillation. An estimation shows that, if the present technique is used in a ground laboratory, the highest viscosity it can measure will be up to $\sim 130 \mathrm{mPa}$. For such high viscosity liquids, a new noncontact technique needs to be developed. Again, if the same experiment is conducted in a reduced gravity environment, the limiting viscosity can be pushed to a much higher value.

\section{ACKNOWLEDGMENTS}

The authors would like to thank Dr. E. Trinh for his interest in this work and for valuable discussions, S. K. Chung and D. Barber for their help, Dr. A. J. Rulison for his help in an early stage of this work, and Eugene Rhim for his proofreading of this manuscript. This work was carried out at the Jet Propulsion Laboratory, California Institute of Technology, under a contract with the National Aeronautics and Space Administration.
${ }^{1}$ T. Iida and R. I. L. Guthrie, The Physical Properties of Liquid Metals (Oxford Science, Clarendon, Oxford, 1988).

${ }^{2}$ M. E. Fraser, W.-K. Lu, A. E. Hamielec, and R. Murarka, Metall. Trans. 2, 817 (1971).

${ }^{3}$ H. Soda, A. McLean, and W. A. Miller, Metall. Trans. B 9, 145 (1978).

${ }^{4}$ B. J. Keene, K. C. Mills, A. Kasama, A. McLean, and W. A. Miller, Metall. Trans. B 17, 159 (1986).

${ }^{5}$ Lord Rayleigh, Proc. R. Soc. London 29, 71 (1879).

${ }^{6}$ D. L. Cummings and D. A. Blackburn, J. Fluid Mech. 224, 395 (1991).

${ }^{7}$ W. K. Rhim, S. K. Chung, D. Barber, K. F. Man, G. Gutt, A. Rulison, and R. E. Spjut, Rev. Sci. Instrum. 64, 2961 (1993).

${ }^{8}$ J. Q. Feng and K. V. Beard, Proc. R. Soc. London, Ser. A 430, 133 (1990).

${ }^{9}$ H. Lamb, Hydrodynamics, 6th ed. (Cambridge University Press, Cambridge, 1932), pp. 473-639.

${ }^{10}$ Lord Rayleigh, Philos. Mag. 14, 184 (1882).

${ }^{11}$ P. M. Adornado and R. A. Brown, Proc. R. Soc. London, Ser. A 389, 101 (1983).

${ }^{12}$ A. Kasama, T. Iida, and Z. Moritar, J. Jpn. Inst. Met. 40, 1030 (1976).

${ }^{13}$ B. J. Keene, Int. Mater. Rev. 38, 157 (1993).

${ }^{14}$ T. Iida, Z. Morita, and S. Takeuchi, J. Jpn. Inst. Met. 11, 1169 (1975).

${ }^{15}$ B. C. Allen, Liquid Metals: Chemistry and Physics, edited by S. Z. Beer (Dekker, New York, 1972), p. 184; also see p. 134 of Ref. 1.

${ }^{16}$ M. G. Frohberg, M. Roesner-Kuhn, and G. Kuppermann, International Workshop on Nucleation and Thermophysical Properties of Undercooled Melts, March 4-6 1998, Physikzentrum Bad Honnef.

${ }^{17}$ S.-F. Chen and R. A. Overfelt, Int. J. Thermophys. 19, 817 (1998). 